The sibbinitted manuscript has been arthored by a contractor of the U.S. Government

under contract No. W-31-109ENG-38.

Accordingly, the U.S. Government retains a

nonexclusive, royalty-free license to publish

of reproduce the publizhed form of this

contribution, of allow others to do $\infty$, for

U. S. Government purposes.

\title{
A Comparison of an Elliptical Multipole Wiggler and Crystal Optics for the Production of Circularly Polarized X-rays
}

REGEIVED

JAN 241995

OSTI

\section{J. C. Lang, George Srajer, and Roger J. Dejus}

Experimental Facilities Division, APS

June 19, 1995

Advanced Photon Source
Argonne National Laboratory

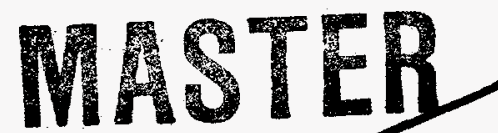

DISTRIBUTION OF THLS DOCWMENT IS UNLRMTED 


\section{DISCLAMMER}

Portions of this document may be illegible in electronic image products. Images are produced from the best available original document. 


\section{Introduction}

Recently, there has been a great deal of interest in polarization modulated $x$-ray diffraction and spectroscopy techniques. ${ }^{[1-3]}$ In particular, the importance of photon helicity in spin-dependent magnetic interactions has expanded the need for high quality circularly polarized $x$-ray sources with fast switching capabilities. Because circularly polarized photons couple differently with the magnetic moment of an atom than do neutrons, they are able to provide unique magnetic information not accessible by neutron techniques. The development of experiments utilizing circularly polarized $x$-rays, however, has been hampered by the lack of efficient sources.

Two different approaches for the production of circularly polarized $x$-rays have attracted the most attention; i) employing specialized insertion devices, and ii) utilizing $x$-ray phase retarders based on perfect crystal optics. For soft $x$-rays (0.1-3.0 keV), source development has centered primarily on insertion devices because there are currently no crystal or multilayer polarizing optics available that cover that full energy range. For harder $x$-rays $(>3.0 \mathrm{keV})$, however, phase retarding optics have been demonstrated, but whether these optics or insertion devices provide the most efficient circularly polarized $x$-ray source in this energy regime has remained a matter of contention. Advocates of each method have made qualitative statements about their advantages, i.e., insertion devices provide a larger flux and phase retarders provide a higher degree of circular polarization, yet a detailed quantitative comparison has been lacking. In this paper, we attempt to provide such a comparison by examining the efficiencies of an elliptical multipole wiggler (EMW) and a standard undulator followed by phase retarding crystal optics. This is done for two different energy regimes, low energies (3.5-13 keV), corresponding to most of the absorption edges of interest 
in resonant scattering and dichroism, and high energies (50-100 keV), which are of interest for magnetic Compton studies and nonresonant magnetic diffraction.

Both insertion devices and phase retarders have been designed and built for beamlines at the Advanced Photon Source (APS). To determine which technique provides the most "efficient" source of circularly polarized $x$-rays, several factors need to be considered. Foremost, due to the inherently small nature of the magnetic $x$-ray cross section, the source should provide both the highest possible flux $(I)$ and degree of circular polarization $\left(P_{c}\right)$. Keep in mind, however, that when comparing two sources the quantity to be maximized is the ratio of the magnetic signal to the charge scattering background. This is normally expressed as the difference between two spectra taken with the opposite helicity or sample magnetization, divided by their sum. Minimizing the error in this quantity requires maximizing the product $P_{c} \cdot \sqrt{I}$ (see appendix). It is this quantity that defines the figure of merit, i.e., the amount of time required to obtain data of comparable quality, when comparing two circularly polarized sources. Furthermore, the source should also be inherently stable, because these measurements generally involve differences in two spectra on the order of $0.1 \%$ and thus are very sensitive to energy shifts and polarization changes. Likewise, the ability to rapidly and frequently reverse the photon helicity is desirable, to avoid systematic errors arising from drift in the beam or experimental apparatus. Finally, the cost of the device for the benefit obtained should be considered as well.

In the following, brief descriptions of the EMW and phase retarding optics are given in sections 2 and 3, respectively. In section 4 , the performance of a lowenergy ( 3.5 to $13 \mathrm{keV}$ ) diamond transmission phase retarder in combination with an undulator is compared to focused and unfocused EMW radiation. In section 
5 , this is extended to the high-energy regime ( 50 to $100 \mathrm{keV}$ ) by utilizing a $\mathrm{Ge}$ Bragg-Laue phase retarder. Our conclusions are summarized in section 6.

\section{Elliptical Multipole Wiggler}

Specialized insertion devices produce circularly polarized $\mathbf{x}$-rays by altering the orbit of the particle beam. In a standard planar insertion device, the particle beam oscillates horizontally producing linearly polarized light on axis. Unlike a bending magnet, however, the off-axis radiation of a planar device is not circularly polarized, because the equal number of left- and right-handed bends in the particle orbit produce equal amounts of left- and right-handed circular polarization resulting in a zero net helicity. In an EMW, a periodic horizontal component to the magnetic field is added, giving the particle beam oscillation a vertical component. This deflects the radiation emitted by the left(right-)handed bends up (down) by an amount $\mathrm{K}_{\mathrm{x}} / \gamma$, where $\mathrm{K}_{\mathrm{x}}$ is the horizontal deflection parameter. Therefore, by looking on-axis of an EMW, one effectively observes the "off-axis" component of each bend. Further, because these are the opposite "off-axis" components for the right- and left-handed bends, the resultant emitted radiation combines to produce circularly polarized photons of a distinct helicity. These devices when coupled with a low-emittance ring, such as the APS, can provide a high flux with a well-defined degree of circular polarization $\left(P_{c} \approx 0.9\right)$ but can suffer from depolarizing effects in the downstream optics.

The APS EMW is based on a design by Gluskin et al.,[4] with the parameters used for the device in this calculation given by Montano et al.[5] The vertical component of the magnetic field is produced by a 37-pole permanent magnet structure made of $\mathrm{NdFeB}$ with a peak field strength of $0.96 \mathrm{~T}$, corresponding to a vertical deflection parameter $K_{y}$ of 14.3. A 36-pole 
electromagnetic structure provides a peak horizontal field of $0.076 \mathrm{~T}$ corresponding to a horizontal deflection parameter $\mathrm{K}_{\mathrm{x}}$ of 1.1. The electromagnet is constructed from laminated iron, allowing for switching frequencies up to 10 $\mathrm{Hz}$. The period of both magnetic structures is $16 \mathrm{~cm}$ making the overall length of the device $2.8 \mathrm{~m}$.

\section{Phase Retarding Optics}

Phase retarders employ perfect crystal optics to transform linear to circular polarization by inducing a $\pm \pi / 2$ phase shift between equal amounts of incoming $\sigma$ and $\pi$ polarized radiation. Being the final optical element before the experiment, they offer the greatest degree of circular polarization incident on the sample $\left(P_{c} \geq 0.9\right)$. The type of phase retarder utilized depends on the energy range of interest. For low energies (3-30 keV) phase retarders which operate in a transmission[6-10] or Bragg reflection ${ }^{[11,12]}$ geometry must be used, while high energies $(>30 \mathrm{keV})$ require phase retarders based on the Laue reflection geometry.[1,13,14] For this comparison, however, only transmission phase retarders are considered at low energies, since Bragg reflection phase retarders offer limited flux and are very sensitive to energy shifts.

\subsection{Low-Energy Transmission Phase Retarders}

In a transmission phase retarder, a thin crystal is deviated a fixed amount $(\Delta \theta \sim 10-100$ arcsec) from the exact Bragg condition and the transmitted beam is used as the circularly polarized x-ray source (Fig. 1). The advantage of this approach is that the polarization properties on the tails of the diffraction peak change relatively slowly as a function of the incoming angle compared to the maxima. Thus the degree of collimation in the incoming beam and the degree of crystalline perfection in the phase retarder required to obtain a well-defined 


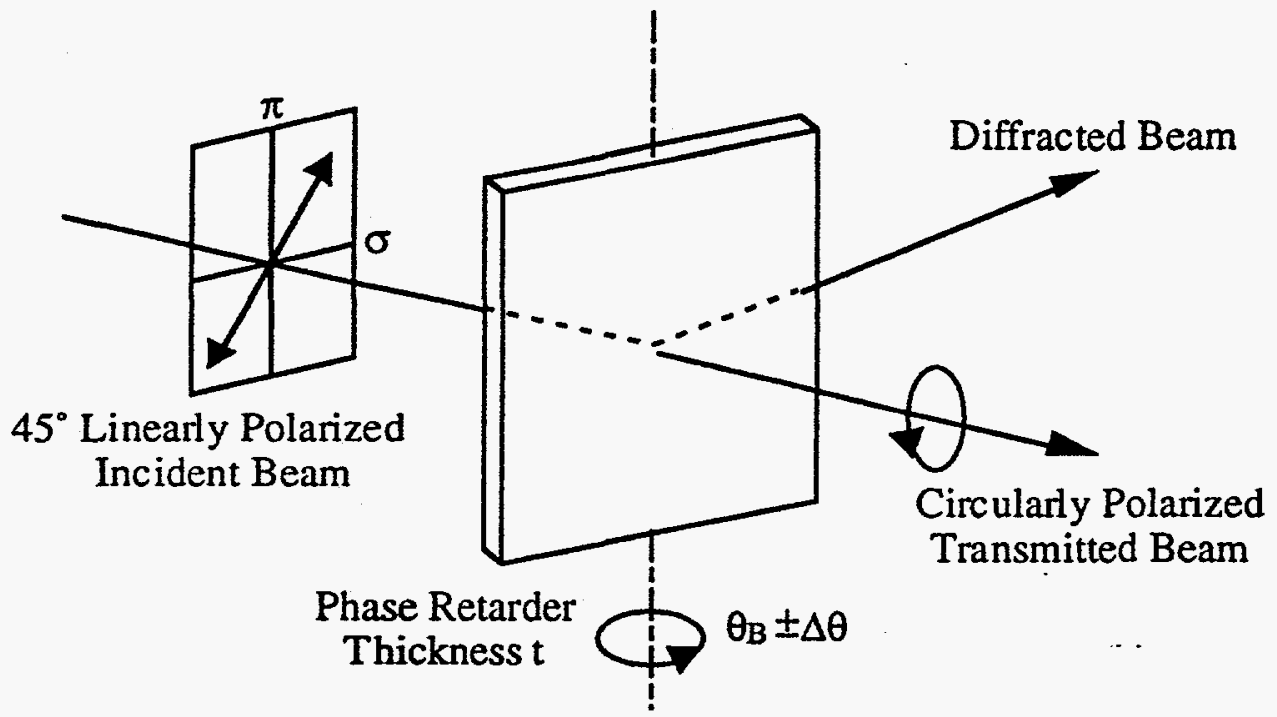

Figure 1 Transmission phase retarder.

polarization state is greatly relaxed compared with phase retarders that require operation at the exact Bragg condition.

Using dynamical diffraction theory, ${ }^{[15]}$ the induced phase lag, $\delta$, for this phase retarder can be expressed in terms of $\Delta \theta$, the deviation from the exact Bragg condition,

$$
\delta=\frac{\pi}{2} \Gamma^{2} \frac{t \sin 2 \theta_{B}}{\lambda \Delta \theta} \operatorname{Re}\left(F_{H} F_{\bar{H}}\right), \quad \Gamma=\frac{r_{e} \lambda^{2}}{\pi V} .
$$

Here $\lambda$ is the wavelength, $t$ is the thickness of crystal traversed by the beam, $\theta_{B}$ is the Bragg angle, $F_{H}$ is the structure factor of the reflection, $V$ is the unit cell volume, and $r_{e}$ is the classical electron radius. Notice that, for a particular crystal thickness and photon energy, the parameter $\Delta \theta$ can be adjusted to obtain a $\pi / 2$ phase shift and $\pm \Delta \theta$ results in $\pm \delta$. The degree of circular polarization in the transmitted beam can be expressed in terms of the phase difference $\delta$ and the $\sigma$ and $\pi$ transmitted field amplitudes, by

$$
P_{c}=\frac{2 E_{\sigma} E_{\pi}}{\left|E_{d}\right|^{2}+\left|E_{\pi}\right|^{2}} \sin \delta,
$$


where $\sigma$ and $\pi$ define the directions perpendicular and parallel to the scattering plane respectively. Therefore, for equal amounts of transmitted $\sigma$ and $\pi$ intensities, a single thin crystal can be used to obtain a nearly total circular polarization at any energy and the helicity can be reversed by simply reversing $\Delta \theta$. Further, this helicity reversal can be accomplished rapidly and frequently because it involves a movement of only a few arc seconds. Recently switching capabilities up to $100 \mathrm{~Hz}$ have been demonstrated for this type of phase retarder. ${ }^{[8]}$ Finally, this degree of polarization is achieved with a minimal attenuation of the $x$-ray beam since reasonable $\Delta \theta$ values, i.e., far enough away from the Bragg reflection, require thicknesses only 1-2 absorption lengths.

In Fig. 2, we show a calculation ${ }^{[16]}$ of the predicted degree of circular polarization as a function of $\Delta \theta$ for a $375-\mu \mathrm{m}$-thick diamond (111) crystal with a perfectly collimated $45^{\circ}$ linearly polarized (with respect to the scattering plane)

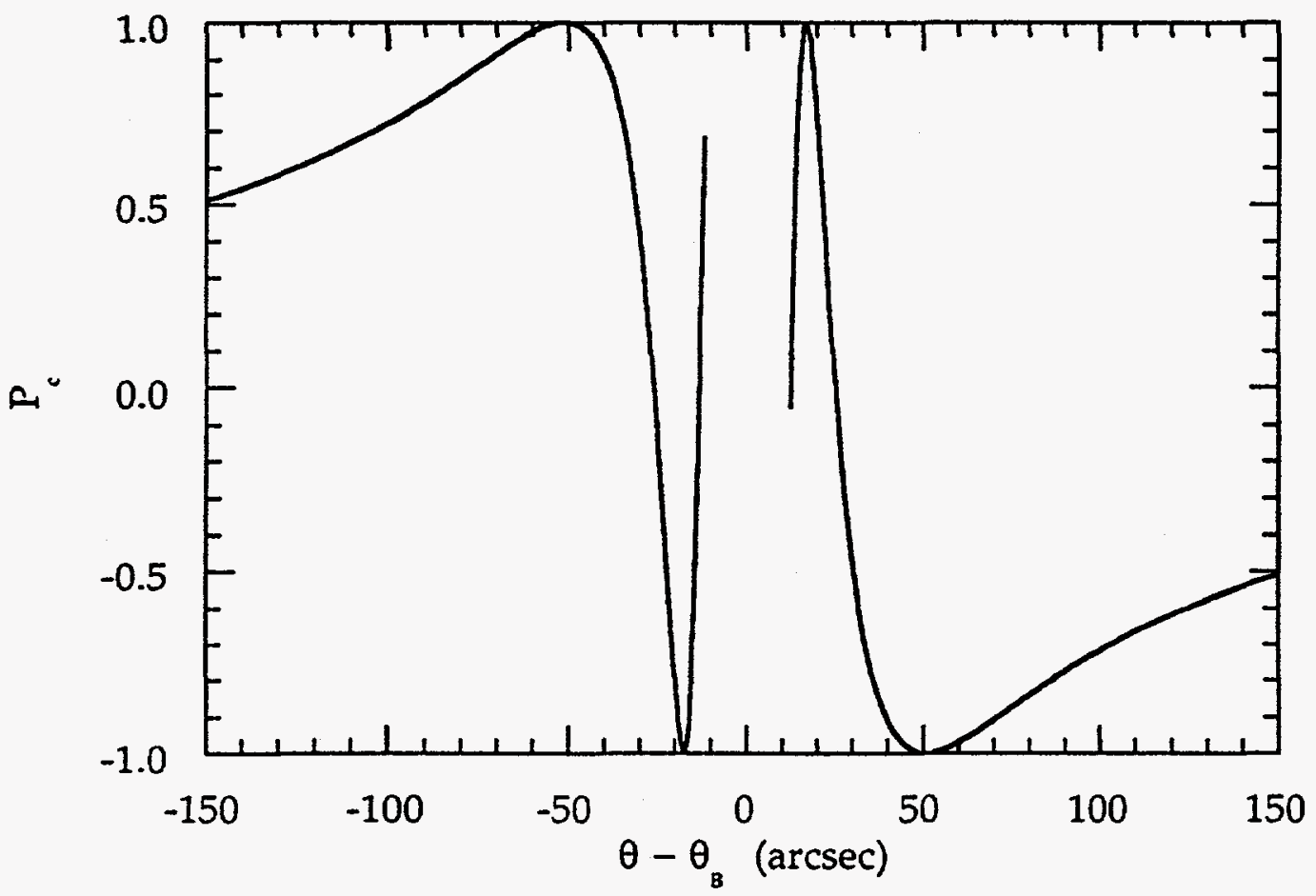

Figure 2 Calculated degree of circular polarization of the transmitted beam for a $375-\mu \mathrm{m}$-thick diamond (111) Bragg reflection with a $8.0 \mathrm{keV} 45^{\circ}$ linearly polarized incident beam. 
$8.0 \mathrm{keV}$ incoming $x$-ray beam. This figure shows that $P_{c}$ is relatively insensitive to angle near $\Delta \theta \approx \pm 50$ arcsec where $P_{c} \approx \pm 1.0$. Therefore beam divergence does not dramatically affect the polarization properties of the transmitted beam. In fact, a 0.5-mrad horizontal divergence from a bending magnet or wiggler radiation fan only reduces $P_{c}$ from \pm 1.00 to \pm 0.86 assuming perfect incoming linear polarization. When utilized with an undulator divergence, as we shall demonstrate, the polarization properties are essentially unaffected. This same insensitivity of $P_{c}$ to the incoming angle greatly relaxes the degree of perfection required in the transmission crystal, with mosaic broadenings 3-4 times the intrinsic rocking curve width not seriously diminishing the performance of the phase retarder.

The optimal choice of crystal, reflection, and energy range for this phase retarder is best seen by setting $\delta=\pi / 2$ and rewriting eq. 1 in the following form,

$$
\frac{\Delta \theta_{c}}{\mu d}=\frac{r_{e}^{2} \operatorname{Re}\left(F_{H} F_{\bar{H}}\right) \lambda^{3}}{\pi^{2} V^{2} \mu} \sin 2 \theta_{B},
$$

where $\mu$ is the linear absorption coefficient. This quantity defines the deviation from the exact Bragg condition required to obtain the maximum circular polarization ( $\Delta \theta_{c}$ ) per absorption length. A plot of eq. 3 for the diamond (111) Bragg reflection is shown in Fig. 3. In order to minimize the effects of beam divergence, $\Delta \theta_{c}$ should be as large as possible while keeping the absorption small; thus for optimum conditions, eq. 3 should be maximized. Therefore, noting that $\mu \sim Z^{4}$ and $F_{H} \sim Z$, where $Z$ is the atomic number, we see that the left hand side of eq. 3 is proportional to $Z^{-2}$ and low- $Z$ materials, such as diamond, $\mathrm{Be}$, or $\mathrm{LiF}$, provide the most suitable phase retarding materials. This equation also demonstrates that this type of phase retarder is limited to low energies 


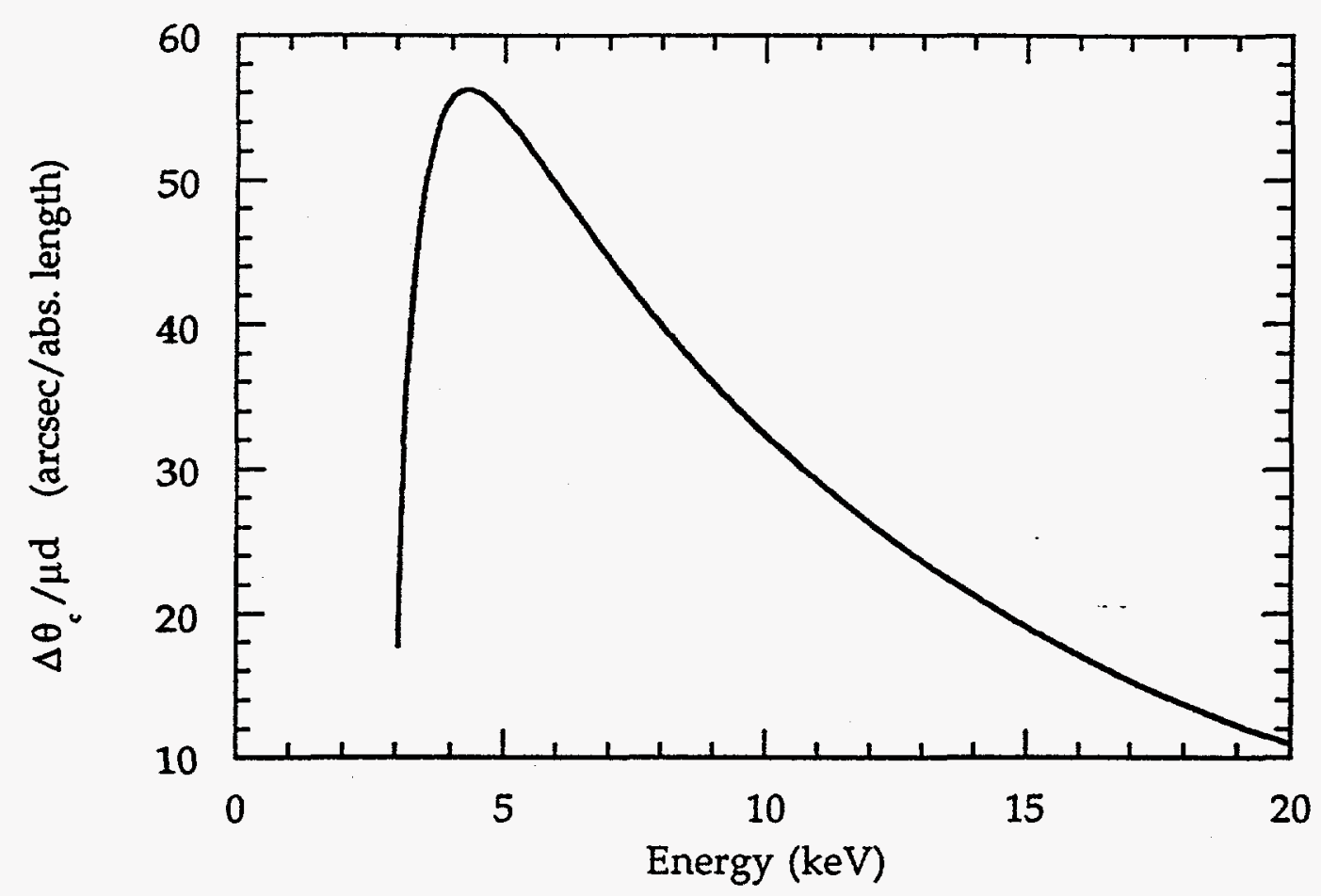

Figure 3 Off-Bragg position required to achieve maximum circular polarization per absorption length for diamond (111) Bragg reflection.

where $\mu \sim \lambda^{3}$ making $\Delta \theta / \mu d \sim \lambda$. At higher energies the increased incoherent cross section makes $\mu \sim \lambda$ and $\Delta \theta / \mu \mathrm{d} \sim \lambda^{3}$, making $\Delta \theta_{\mathrm{c}}$ rapidly go to zero above $30 \mathrm{keV}$.

\subsection{High-Energy Laue Reflection Phase Retarder}

For energies greater than $30 \mathrm{keV}$, a phase retarder based on a Laue reflection must be used. On the Bragg condition, the phase lag between the $\sigma$ and $\pi$ wave fields of the $\alpha$-branch of the dispersion surface is given by,

$$
\delta=\Gamma F_{H} \frac{t \pi\left(1-\mid \cos 2 \theta_{B}\right)}{\lambda}, \Gamma=\frac{r_{e} \lambda^{2}}{\pi V}
$$

Eq. 4 indicates that this phase retarder can only yield $\pi / 2$ phase shifts at discrete energies determined by the thickness $t$. This limitation, however, is not a serious flaw because experiments in this energy regime generally do not involve energy 
scanning. Another drawback with this phase retarder has been its inability to provide for helicity reversal in a convenient fashion, although recently some designs have been proposed to allow for this.[17,18] High- $Z$ materials, such as $G e$, provide the optimum crystals for this phase retarder for two reasons. The phase shift for high- $Z$ materials changes more slowly over the width of the reflectivity curve and the absorption is increased for the $\beta$-branch of the dispersion curve, which induces the opposite phase retardation as the $\alpha$-branch diminishing the obtained $\mathrm{P}_{\mathrm{c}}$.

\section{Comparison for the Low-Energy Regime}

The experimental setups compared for the low-energy regime (3.5-13 keV) are illustrated in Fig. 4. For the phase retarder, $x$-rays were obtained from APS undulator A.[19,20] The highly collimated nature of the undulator beam in both the vertical and horizontal directions $\left(\sigma_{x^{\prime}} \approx 23 \mu \mathrm{rad}, \sigma_{y^{\prime}} \approx 9 \mu \mathrm{rad}\right)$ and the welldefined linear polarization state $\left(P_{1} \approx 0.99\right)$ make this an ideal source for phase retarding optics. The central cone of the undulator radiation was isolated using $2 \times 1 \mathrm{~mm}$ slits positioned $30 \mathrm{~m}$ from the source, with the first harmonic used from $3.5-10 \mathrm{keV}$ and the third harmonic from $10-13 \mathrm{keV}$. The tuning curve spectra were calculated for an ideal magnetic lattice using the US[21] code developed at the APS. The flux obtained from a real device could be reduced due to magnetic imperfections, but recent calculations incorporating the measured undulator magnetic field have shown that the flux obtained from the first and third harmonics is expected to be greater than $95 \%$ that of the ideal case.

The beam was monochromatized by a Si (111) double-crystal monochromator (DCM) with a $90 \%$ detune. Detuning of the monochromator was included in order to accurately reflect normal experimental conditions in energy scanning measurements. The beam was then incident on a diamond (111) 
Si (111)

Monochromator

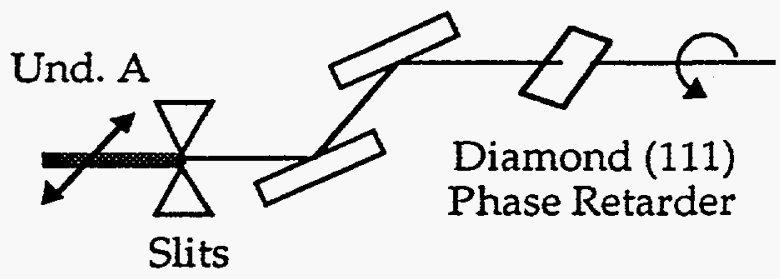

(a)
Sagittal Si (111)

Monochromator

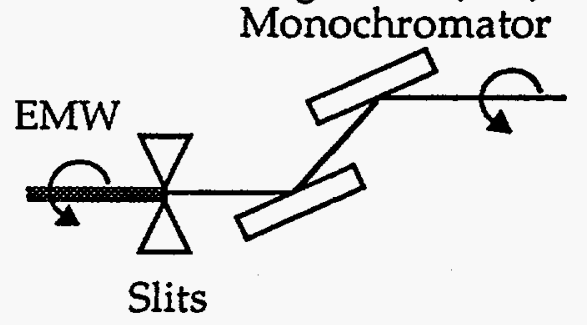

(b)

Figure 4 Schematic of setups for producing circularly polarized $x$-rays in the low energy regime. a) $2 \times 1 \mathrm{~mm}$ linearly polarized incident undulator beam, Si (111) monochromator, and 1.5-abs. length diamond (111) Bragg reflection phase retarder. b) EMW elliptically polarized incoming beam, $\mathrm{Si}$ (111) monochromator, i) $2 \times 1 \mathrm{~mm}$ unfocused beam, ii) $12 \times 1 \mathrm{~mm}$ and $60 \times 1 \mathrm{~mm}$ sagittally focused beams.

Bragg transmission phase retarder with the diffraction plane oriented at $45^{\circ}$ to the synchrotron orbit, in order to provide equal amounts of incoming $\sigma$ and $\pi$ polarization. This reflection was chosen because it allowed access to energies down to $\sim 3.0 \mathrm{keV}$. While no crystal possesses a constant attenuation over the entire energy range considered, a set of five different diamond thicknesses can provide crystals varying from 1 to 2 absorption lengths. Therefore, the calculations of the resultant flux were performed assuming a constant 1.5-abs. length attenuation for the phase retarder. The polarization of the transmitted beam was obtained by convoluting the polarization profile (Fig. 2) of the phase retarder with the undulator divergence at each step in energy.

Spectra of the emitted EMW radiation were calculated using the WS[21] code for slit sizes of $2 \times 1 \mathrm{~mm}, 12 \times 1 \mathrm{~mm}$, and $60 \times 1 \mathrm{~mm}$ positioned $30 \mathrm{~m}$ from the source, with the deflection parameters set to $K_{y}=14$ and $K_{x}=1$. While these calculations did not include the particle beam emittance, this does not seriously impact the results obtained for the flux and polarization, since the angle 
subtended by the vertical slit size $\left(\theta_{y} \approx 33 \mu \mathrm{rad}\right)$ is much larger than the beam emittance $\left(\sigma_{y^{\prime}} \approx 9 \mu \mathrm{rad}\right)$. More quantitatively, depolarization is unimportant when the following condition is met, [22]

$$
\mathrm{K}_{\mathrm{x}} \gg \gamma \sqrt{\sigma_{\mathrm{y}^{\prime}}^{2}+\left(\theta_{\mathrm{y}} / 2\right)^{2}}
$$

where $\gamma^{-1}$ is the opening angle of the synchrotron radiation $(\approx 73 \mu \mathrm{rad})$.

The $2 \times 1 \mathrm{~mm}$ slit defined an EMW beam with approximately the same divergence as the undulator. This allowed for direct comparison of the two sources in experiments in which the brilliance of the beam might become important (i.e., looking at thin magnetic films). The $60 \times 1 \mathrm{~mm}$ beam intercepted the full EMW radiation fan and was used to compare the maximum possible flux on sample obtainable by each technique. To minimize the size of the beam at the sample position, the beam was sagittally focused by the second crystal in the DCM for this case. Ideal bending was assumed for the focusing crystal over the entire width of the beam; since this is never the case, we have also included an intermediate $12 \times 1 \mathrm{~mm}$ slit size. Just as for the phase retarder, a Si (111) DCM with a $90 \%$ detune was used. The undulator and EMW calculations were both performed with the ring operating at $7.0 \mathrm{GeV}$ and $100 \mathrm{~mA}$ of current. Attenuation due to the beamline windows was not included for either spectra because this does not affect the comparison between the sources, but should be factored in flux numbers given, especially for lower energies.

The incident flux, degree of circular polarization, and figure of merit $\left(\mathrm{P}_{\mathrm{c}} \cdot \sqrt{\mathrm{I}}\right)$ at the sample position, i.e., after the optical components, for each of these cases are shown in Figs. 5-7. The two lines shown for the phase retarder in Figs. 5 and 7 indicate the first and third harmonic tuning curves. Fig. 5 shows that the phase retarder when utilized with an undulator beam provides a flux 
comparable to that of the fully focused EMW radiation fan and provides a much greater flux than the unfocused $2 \times 1 \mathrm{~mm}$ and focused $12 \times 1 \mathrm{~mm}$ EMW beams. This high flux obtained from the phase retarder is accompanied by a significantly higher degree of circular polarization as shown in Fig. 6. For the undulator divergence, the phase retarder produces $P_{c}$ values ranging from 0.995 at $4.5 \mathrm{keV}$ (the peak in Fig. 3) to 0.975 at $13 \mathrm{keV}$, while the EMW peaks at 0.87 at $13 \mathrm{keV}$. Only one curve is shown for the EMW since the different horizontal slit sizes changed $\mathrm{P}_{c}$ by only $0.5-2 \%$, with the $60 \times 1 \mathrm{~mm}$ beam yielding the highest values. The dramatically lower polarization obtained for the EMW at energies below 6 $\mathrm{keV}$ results from the lower $\pi$ reflectivity as the Bragg angle for the Si (111) monochromator nears 45 degrees, which is accentuated by the detuning. At higher energies, the depolarization from the optics becomes minimal and $P_{c}$ approaches $P_{c} \approx 0.89$ emitted by the EMW before the monochromator.

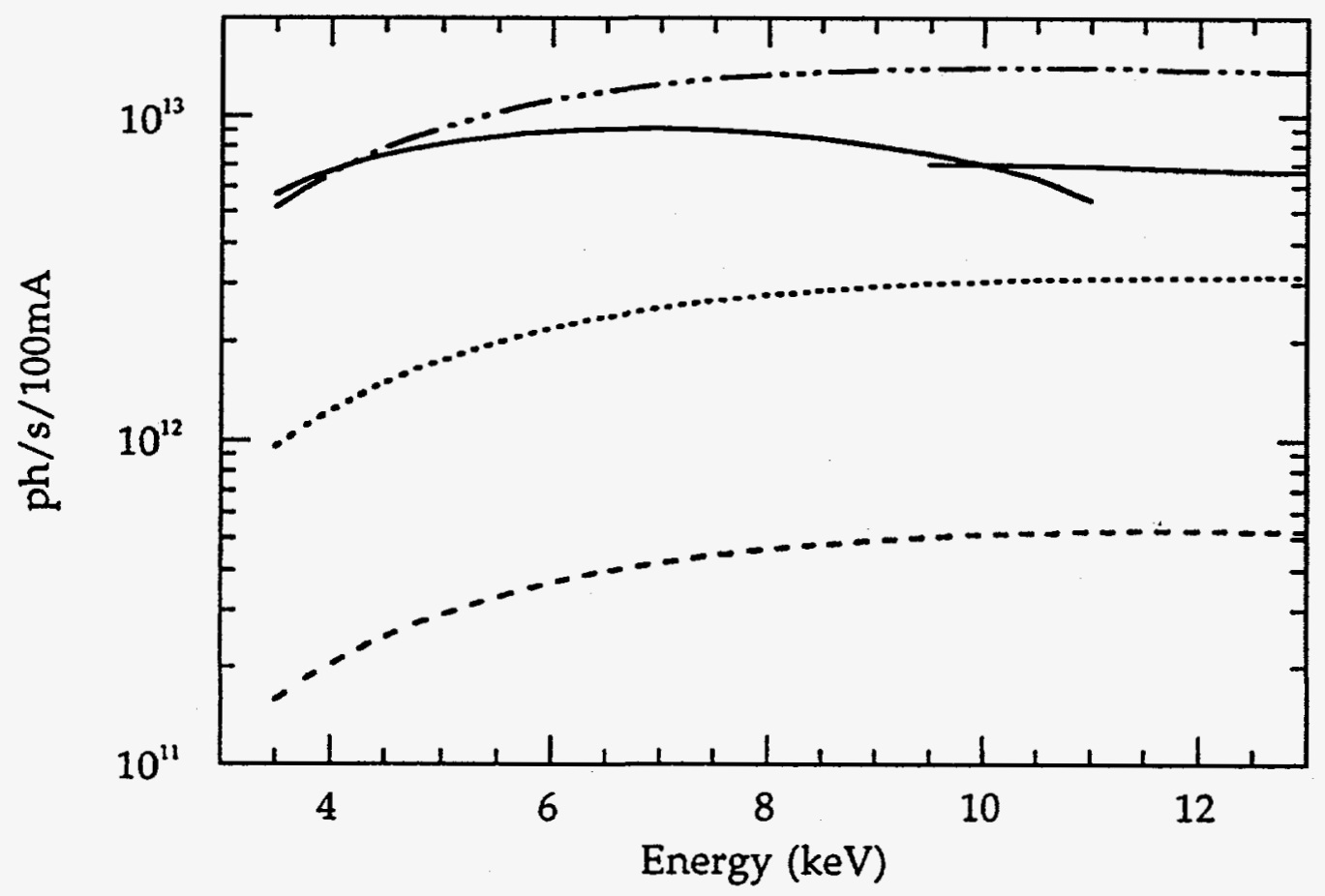

Figure 5 Flux on sample for an undulator source with phase retarder (solid), EMW $2 \times 1 \mathrm{~mm}$ slit unfocused (dashed), $12 \times 1 \mathrm{~mm}$ slit focused (dotted), and $60 \times 1 \mathrm{~mm}$ slit focused (dash-dotted). 


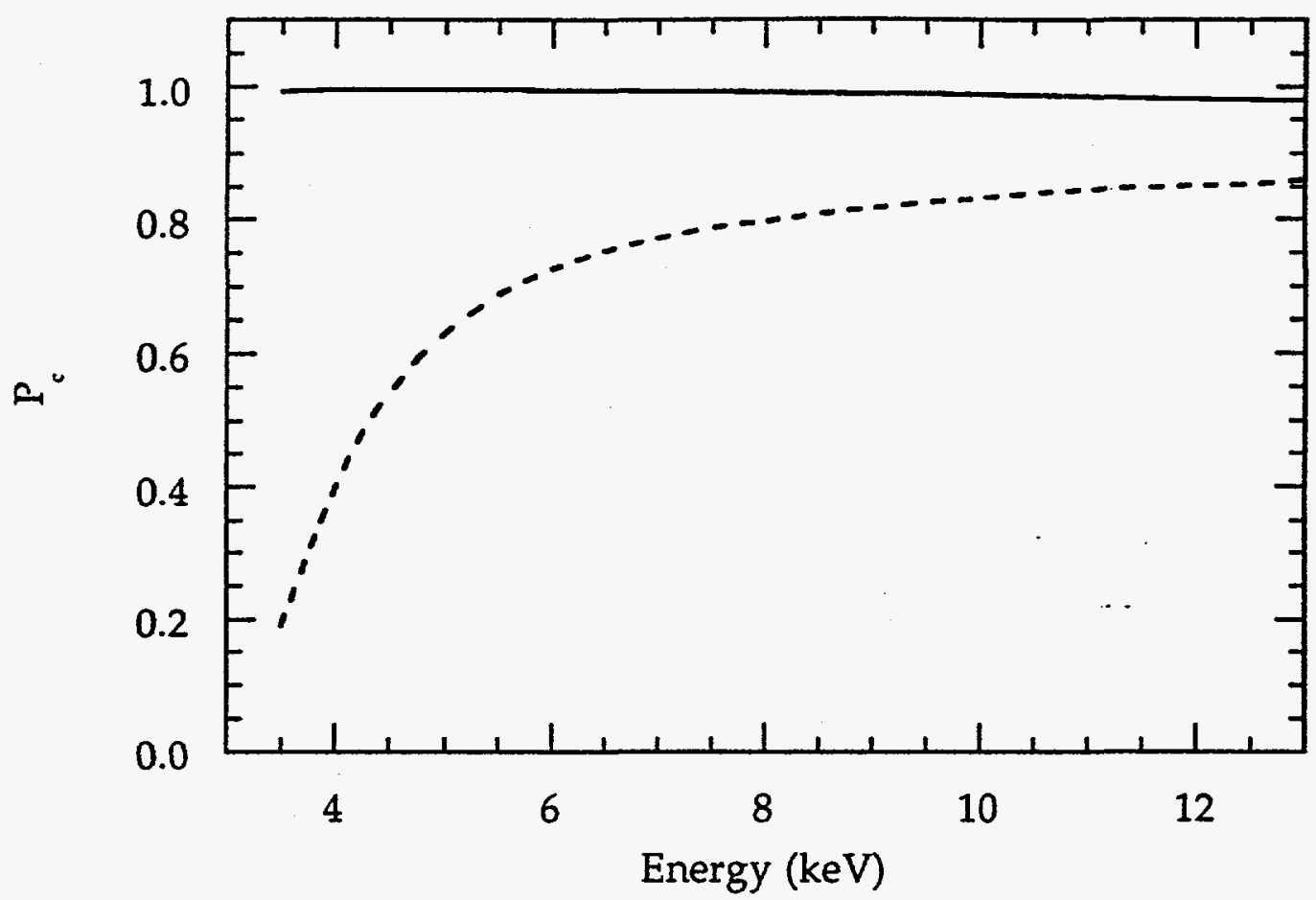

Figure 6 Degree of circular polarization for an undulator source with phase retarder (solid) and EMW (dashed).

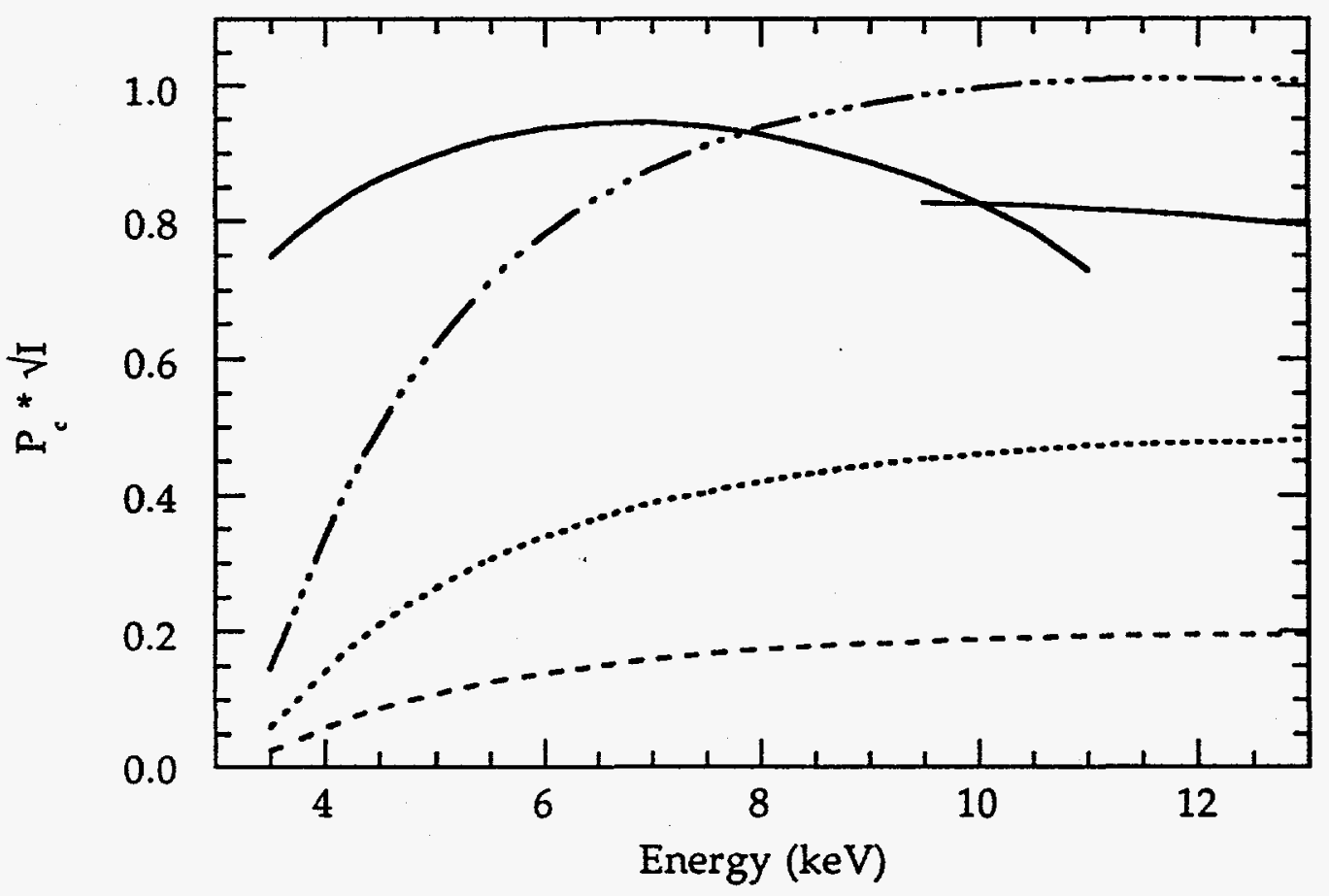

Figure 7 Figure of merit for an undulator source with phase retarder (solid), EMW $2 \times 1 \mathrm{~mm}$ slit unfocused (dashed), $12 \times 1 \mathrm{~mm}$ slit focused (dotted), and $60 \times 1 \mathrm{~mm}$ slit focused (dash-dotted). 
As stated earlier, the true comparison for two circularly polarized sources is the figure of merit shown in Fig. 7, which has been normalized such that 1.0 corresponds to $1 \times 10^{13} \mathrm{ph} / \mathrm{s}$ with $P_{c}=1.0$. This figure demonstrates that, for energies below $8.0 \mathrm{keV}$, the values obtained for the diamond phase retarder with an undulator beam are larger than all cases shown for the EMW beam. The largest difference occurs at $3.5 \mathrm{keV}$ where, due to the depolarization effects from the downstream optics, the phase retarder is a factor of 5 larger than the fully focused EMW beam and 30 larger than the unfocused $2 \times 1 \mathrm{~mm}$ beam. Monochromators which can compensate for the decreased $\pi$ reflectivity at energies below $8 \mathrm{keV}$ have been suggested[23,24] but generally involve four crystal reflections, which increases $P_{c}$ by approximately a factor of 3 at $3.5 \mathrm{keV}$ but reduces the throughput by an order of magnitude compared to a conventional DCM. Therefore the figure of merit for an EMW with this type of monochromator is not significantly better than that shown in Fig. 7. At $8.0 \mathrm{keV}$, the phase retarder and the fully focused EMW beam become comparable, but the phase retarder is still a factor of 2 larger than the $12 \times 1 \mathrm{~mm}$ focused EMW beam and 5 greater than the unfocused beam. For energies above $8.0 \mathrm{keV}$, the full EMW beam yields the larger figure of merit, becoming $20 \%$ larger than the phase retarder and undulator by $13.0 \mathrm{keV}$. We should mention again, however, that we have assumed ideal bending over the full $60 \mathrm{~mm}$ width of the beam in the sagittally focused crystal. In practice, the flux attained from the focused crystal probably is lower, thus the true difference between the two techniques in the 8.0 to $13.0 \mathrm{keV}$ energy range is undoubtedly smaller. This analysis could be extended for energies up to $30 \mathrm{keV}$ using transmission phase retarders, with the observed trends continuing, i.e., the phase retarder should be just below the focused full EMW beam but significantly larger than the unfocused beam of comparable size. Therefore for these energies, the diamond phase retarder in 
combination with an undulator provides a source of circularly polarized $\mathrm{x}$-rays comparable to a fully focused EMW beam, and for brilliance limited experiments the phase retarder is clearly the more efficient source.

\section{Comparison for the High-Energy Regime}

The setups compared for the high-energy regime (50-100 keV) are shown in Fig. 8. Again for the phase retarder, a $2 \times 1 \mathrm{~mm} x$-ray beam obtained from undulator A was utilized. Recent calculations have indicated that undulator A at closed gap ( $10.5 \mathrm{~mm} \mathrm{~K}_{\mathrm{y}}=2.87$ ) can provide significantly higher flux through a modest size pinhole $(<5 \times 2 \mathrm{~mm})$ at these energies than an APS wiggler operating with a $1.0 \mathrm{~T}$ field ( $\left.\mathrm{K}_{\mathrm{y}}=7.87\right) .{ }^{25]}$ When field errors in the magnetic structure of the undulator are considered, much of the harmonic structure of the emitted spectra is washed out at higher energies, providing a fairly uniform source for this energy range. For this calculation, a smooth curve was extrapolated through the remaining structure in the undulator spectra to obtain the emitted flux. The beam was then diffracted by Ge (220) Bragg and Laue reflections. The Laue

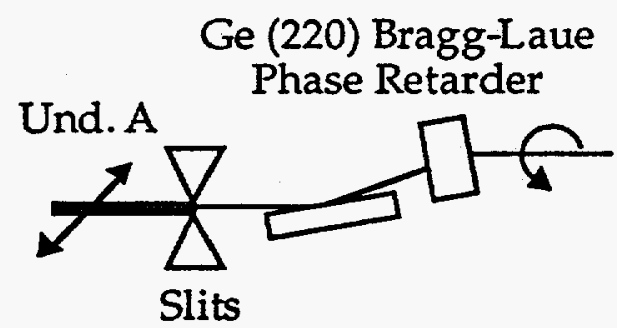

(a)
$\mathrm{Ge}(220)$ Monochromator

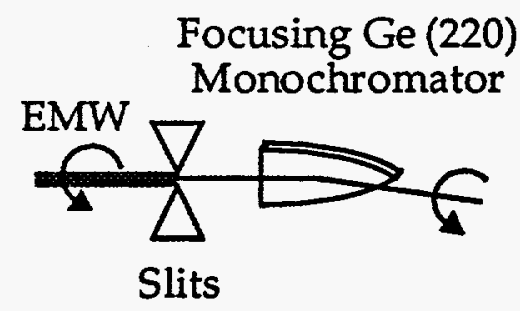

(c)

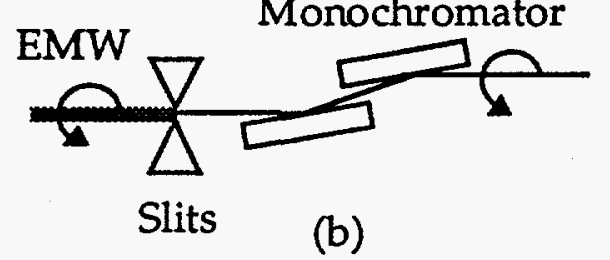

(b)

Figure 8 Schematic of setups for the production of circularly polarized $x$-rays in the high-energy regime. a) $2 \times 1 \mathrm{~mm}$ undulator beam, Ge BraggLaue (220) phase retarder. b) $2 \times 1 \mathrm{~mm}$ EMW beam, Ge (220) DCM. c) $12 \times 1 \mathrm{~mm}$ EMW beam, 1-mm-thick meridinally focused $\mathrm{Ge}$ (220) monochromator diffracting horizontally. 
reflection produced the phase retardation, with its thickness adjusted to produce $\pi / 2$ phase shifts at each energy. Again the scattering plane of the phase retarder was oriented $45^{\circ}$ with respect to the synchrotron orbit in order to obtain equal intensities for the incoming $\sigma$ and $\pi$ components.

The calculated phase retarder spectra were compared with four different cases for the EMW, two different slit sizes $(12 \times 1 \mathrm{~mm}$ and $2 \times 1 \mathrm{~mm}, 30 \mathrm{~m}$ from the source) and two different values for the horizontal deflection parameter $\left(\mathrm{K}_{\mathrm{x}}=0.5\right.$ and $K_{x}=1.0$ ). The reason for the added horizontal deflection parameter comparison in this energy regime is illustrated in Fig. 9, which shows the flux obtained through a $2 \times 1 \mathrm{~mm}$ pinhole for $\mathrm{K}_{\mathrm{x}}=0.0,0.5$, and 1.0. This figure demonstrates that, although the degree of circular polarization goes up as $K_{\mathbf{x}}$ increases, the amount of high-energy flux decreases dramatically. This is because the on-axis beam of the EMW is looking at an "off-axis" component of the deflected radiation lobes from the left- and right-handed bends. At higher

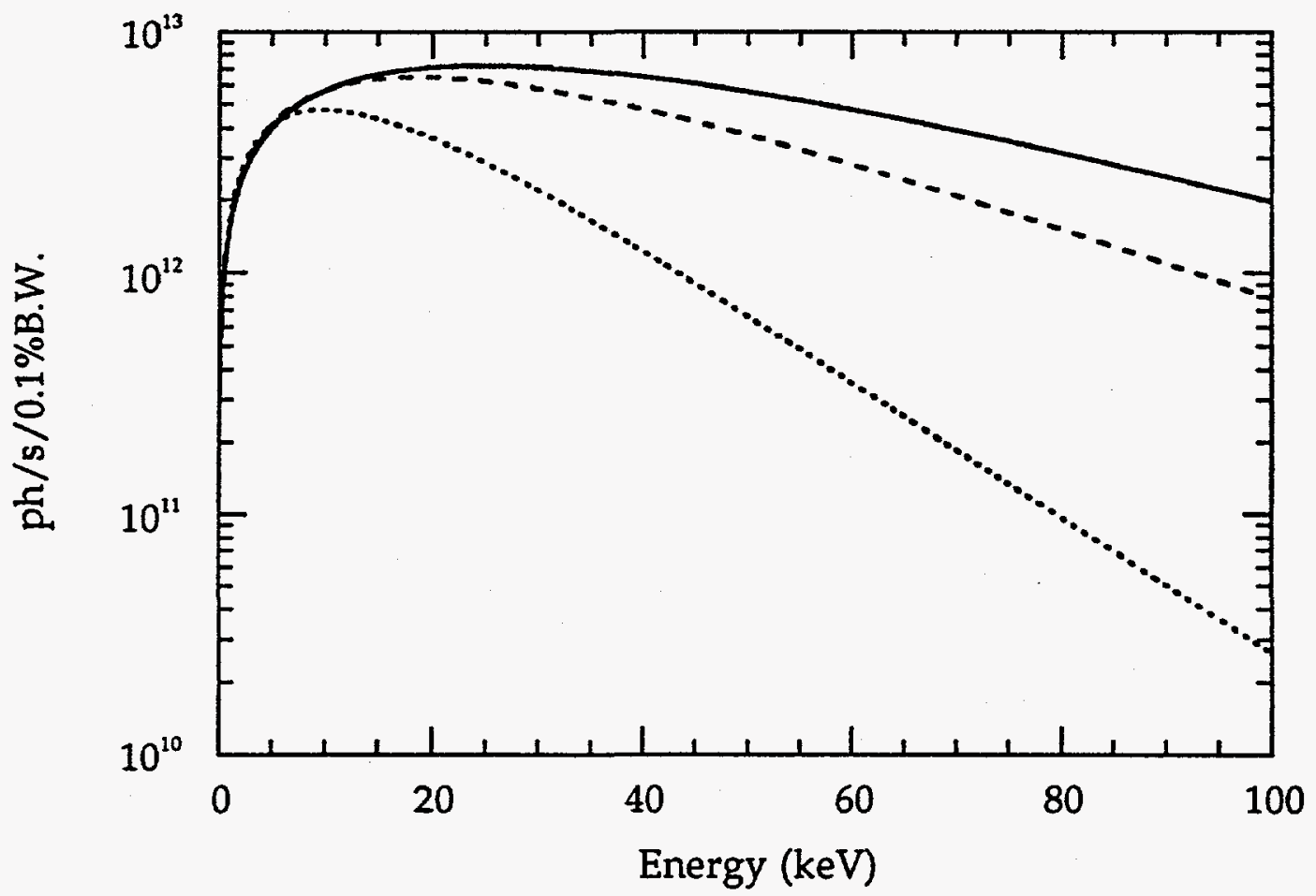

Figure 9. Flux emitted by the EMW through a $2 \times 1 \mathrm{~mm}$ slit for $\mathrm{K}_{\mathrm{x}}=0.0$ (solid), $K_{x}=0.5$ (dashed), and $K_{x}=1.0$ (dotted). 
energies, the radiation becomes much more collimated along these lobes. Thus, when looking on-axis of the EMW, the degree of circular polarization increases but the amount of flux is reduced (i.e., effectively looking further "off-axis" of each lobe). Therefore we have chosen two cases to compare for the EMW in this energy range, $K_{x}=0.5$, which provides a value close to the maximum figure of merit, and $K_{x}=1.0$, which yields the maximum $P_{c}$. For the lower energy comparison, only $\mathrm{K}_{\mathrm{x}}=1.0$ was included since the flux does not decrease as dramatically in this energy range, as indicated by the nearly overlapping curves below $10 \mathrm{keV}$ in Fig. 9. Thus the maximum figure of merit at lower energies is obtained by setting $K_{x}=1.0$ to maximize $P_{c}$.

The downstream optics for the EMW consisted of a conventional Ge (220) $\mathrm{DCM}$ for $2 \times 1 \mathrm{~mm}$ beam and a single horizontally diffracting 1-mm-thick $\mathrm{Ge}$ (220) crystal with meridinal 1:1 focusing for the $12 \times 1 \mathrm{~mm}$ beam. The 12-mm horizontal extent of the beam, which intercepted approximately $20 \%$ of the full width of the EMW fan, was the maximum possible for this focusing scheme due to the large beam footprint at these higher energies. This calculation assumed that the bending did not broaden the rocking curve of the crystal. The effective broadening due to the finite thickness of the crystal, however, was included in the flux calculations.

The calculated flux on sample obtained for the different $K_{x}$ values are shown in Figs. 10 and 11. With $K_{x}=0.5$, Fig. 10, the focused EMW beam provides almost an order of magnitude more flux than the phase retarder over the entire range and the unfocused $2 \times 1 \mathrm{~mm}$ beam is slightly above, but comparable to the phase retarder. For $K_{x}=1.0$, Fig. 11, the flux obtained from the phase retarder and the focused EMW beam are comparable, with the phase retarder actually yielding a higher flux for energies above $67 \mathrm{keV}$. The unfocused beam is down significantly, ranging from approximately a factor of 3 to 20 lower than the phase 


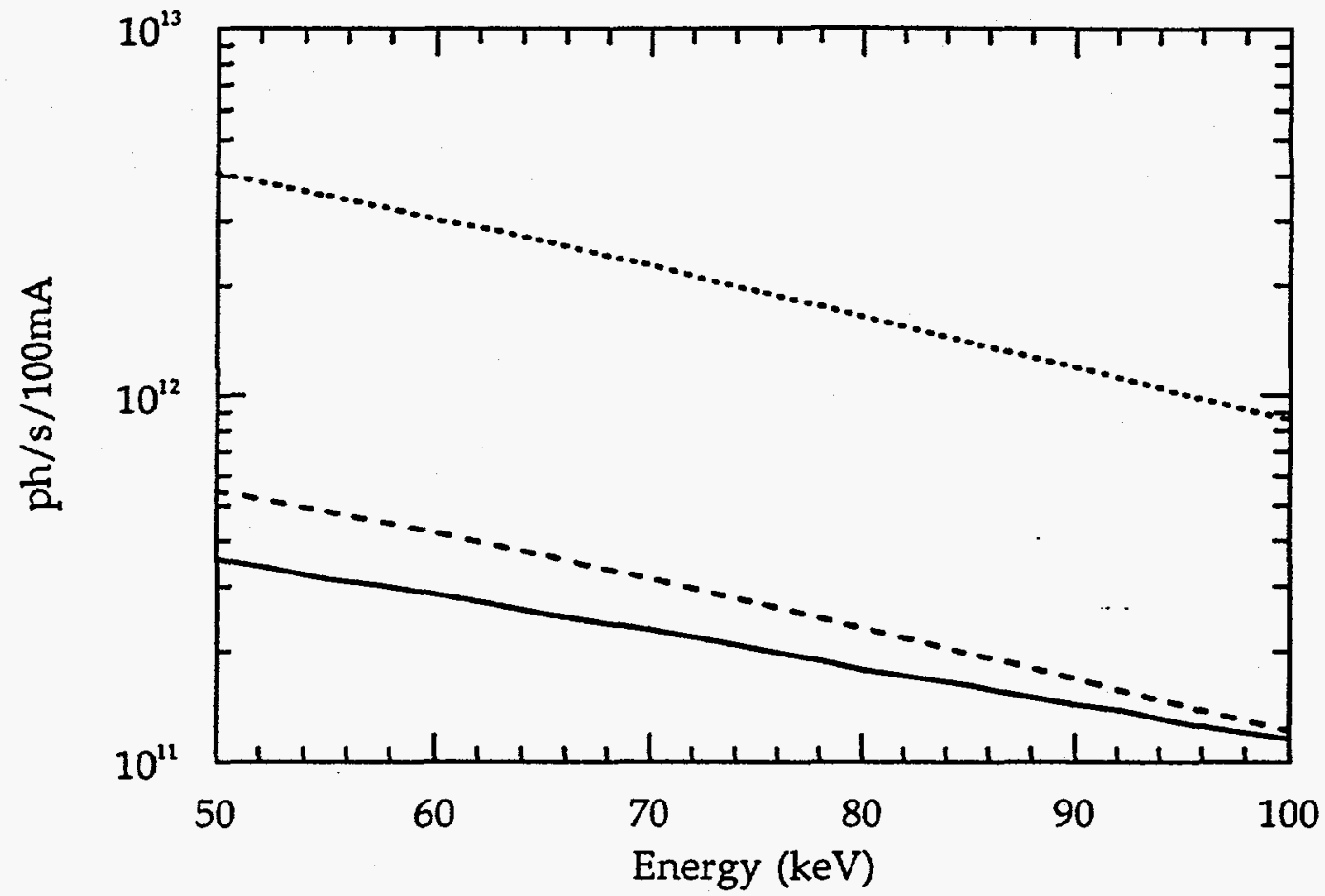

Figure 10 Flux on sample for an undulator source with phase retarder (solid), EMW $\mathrm{K}_{\mathrm{x}}=0.52 \times 1 \mathrm{~mm}$ slit unfocused (dashed), and $12 \times 1 \mathrm{~mm}$ slit focused (dotted).

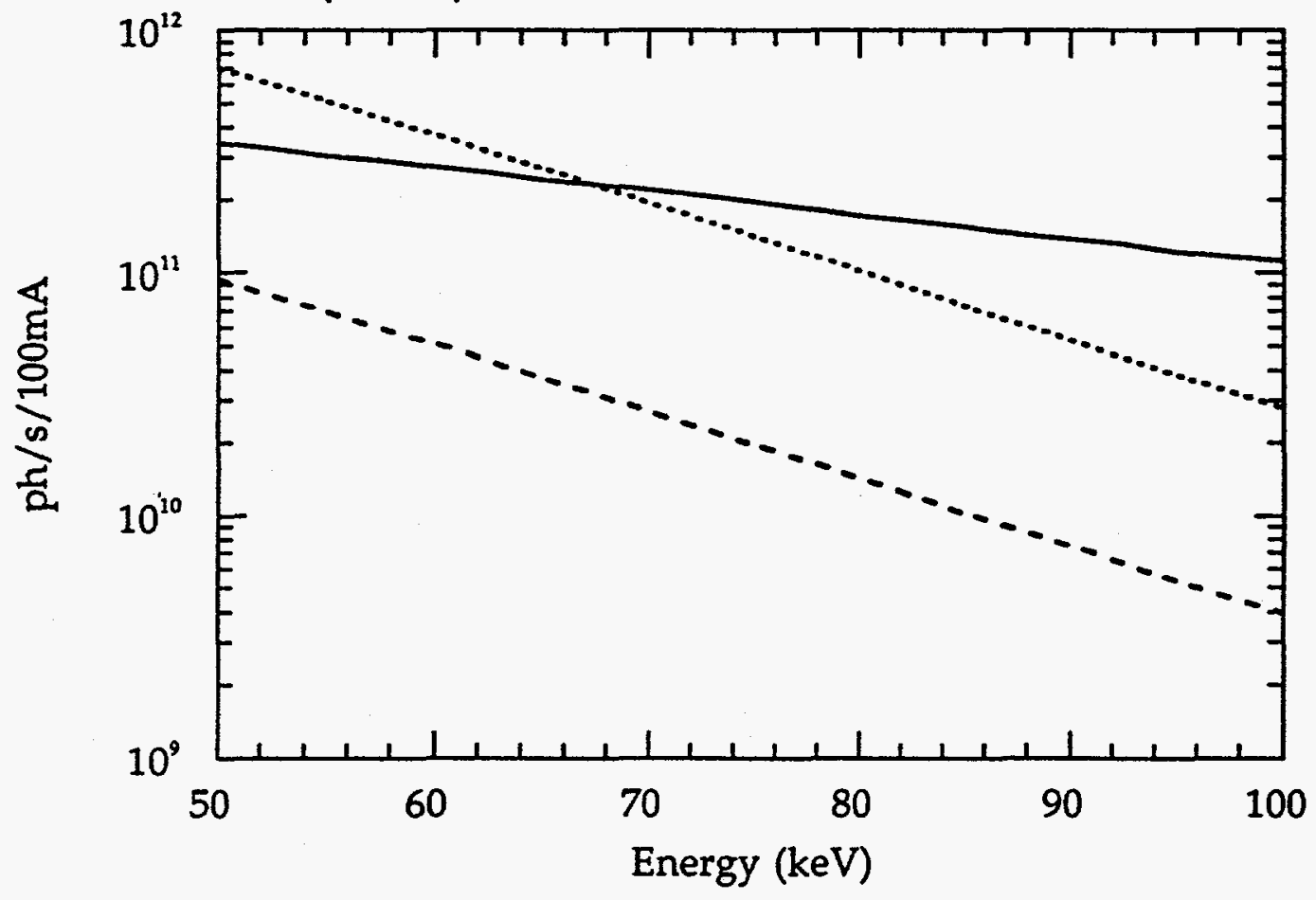

Figure 11 Flux on sample for an undulator source with phase retarder (solid), EMW $\mathrm{K}_{\mathrm{x}}=1.02 \times 1 \mathrm{~mm}$ slit unfocused (dashed), and $12 \times 1 \mathrm{~mm}$ slit focused (dotted). 


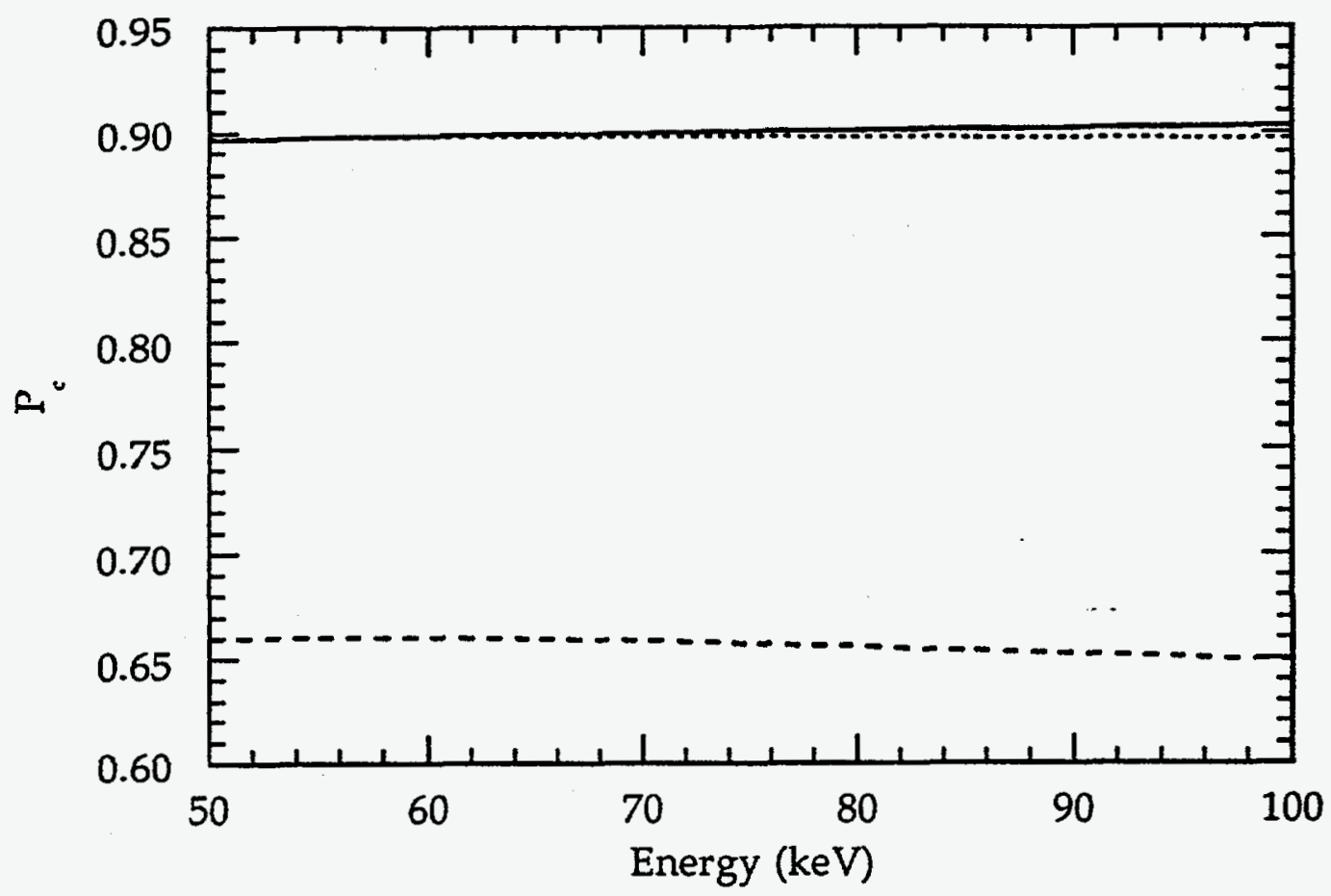

Figure 12 Degree of circular polarization for an undulator source with phase retarder (solid), EMW $K_{x}=0.5$ (dashed), and EMW $K_{x}=1.0$ (dotted).

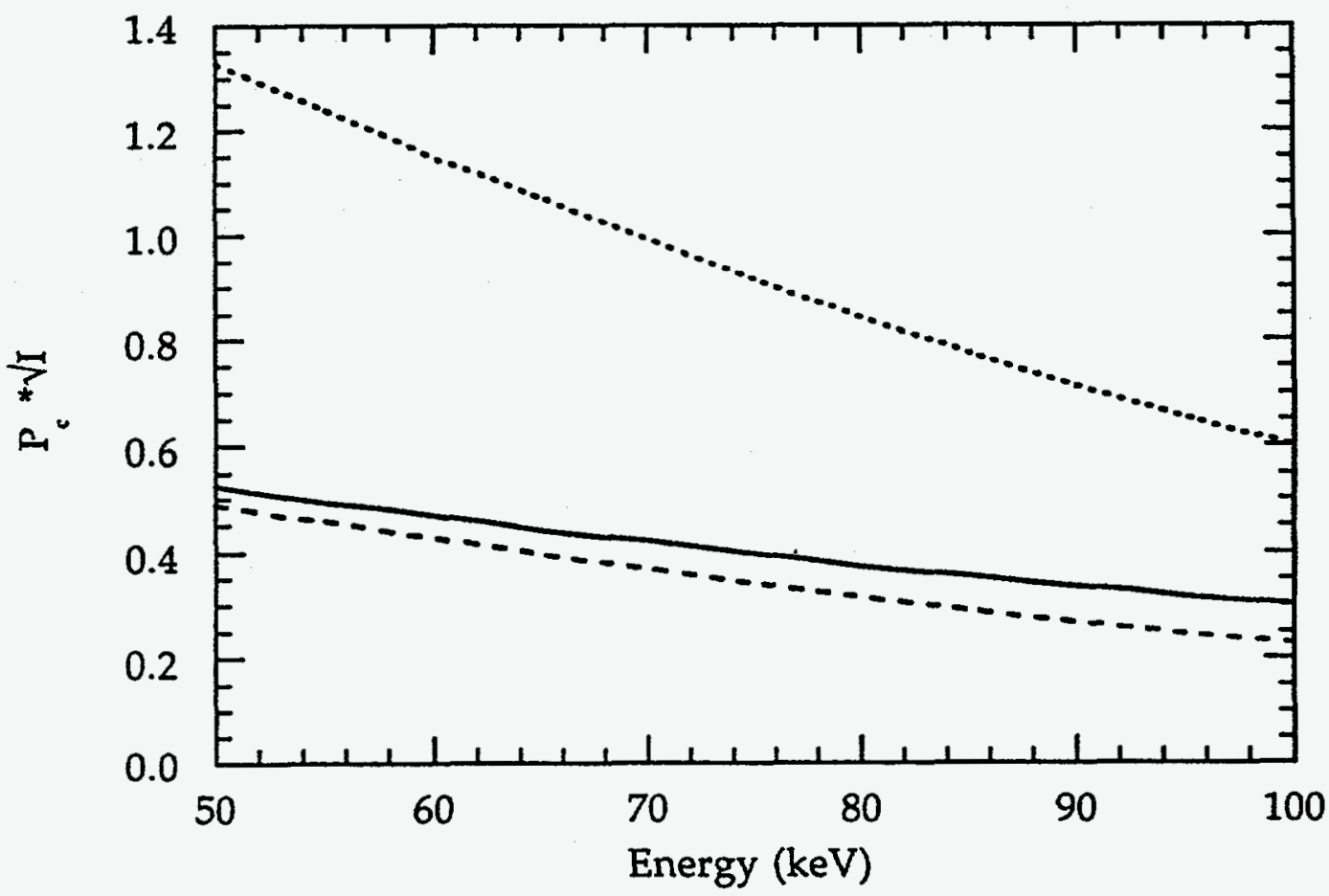

Figure 13 Figure of merit for an undulator source with phase retarder (solid), EMW $\mathrm{K}_{\mathrm{x}}=0.52 \times 1 \mathrm{~mm}$ slit unfocused (dashed), and $12 \times 1 \mathrm{~mm}$ slit focused (dotted). 


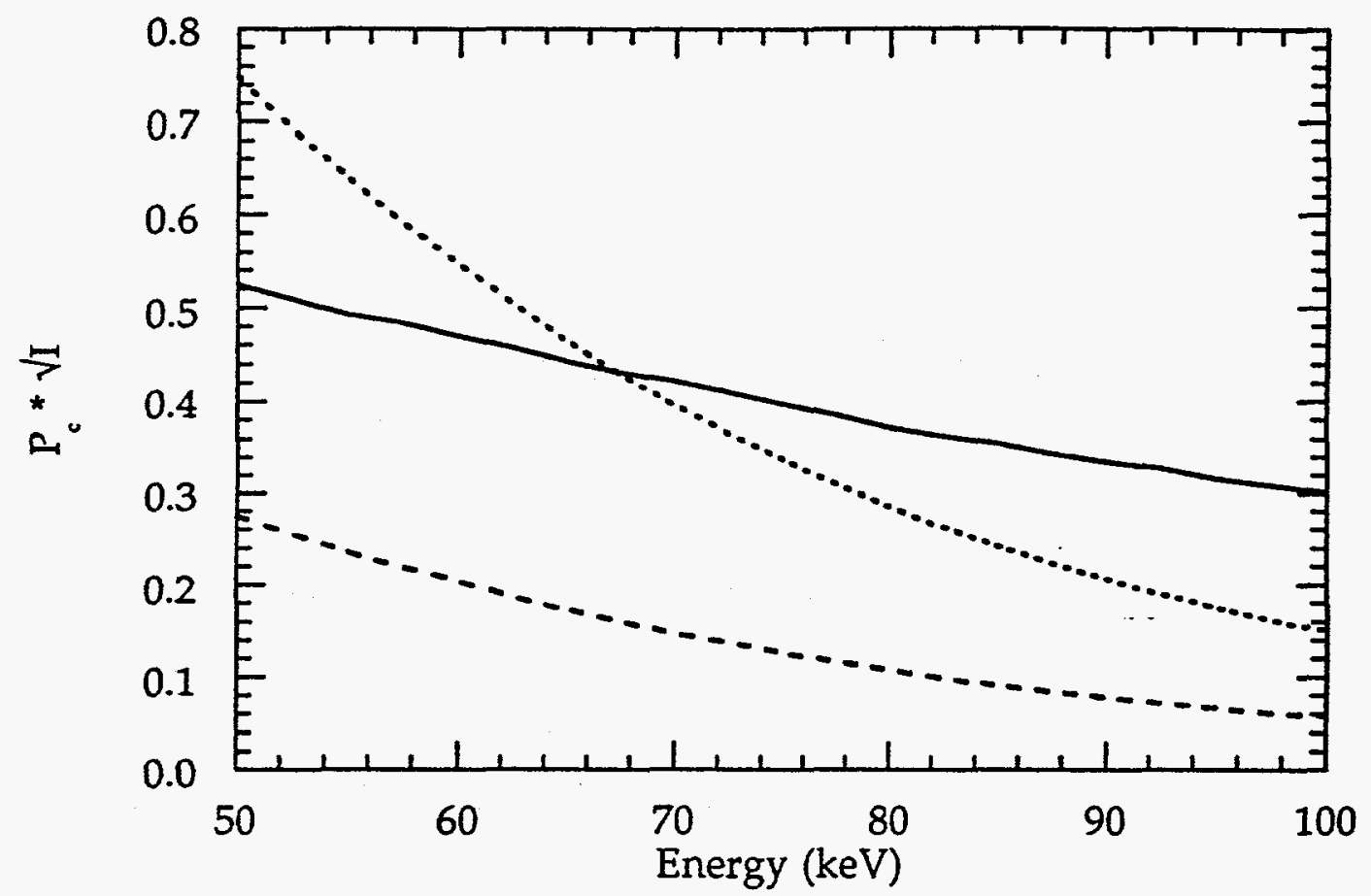

Figure 14 Figure of merit for an undulator source with phase retarder (solid), EMW $\mathrm{K}_{\mathrm{x}}=1.02 \times 1 \mathrm{~mm}$ slit unfocused (dashed), and $12 \times 1 \mathrm{~mm}$ slit focused (dotted).

retarder. The degree of circular polarization obtained for the phase retarder and the EMW at $K_{x}=0.5$ and 1.0 are shown in Fig. 12. The $P_{c}$ values obtained for the phase retarder and the EMW at $\mathrm{K}_{\mathrm{x}}=1.0$ are approximately 0.90 while the EMW at $K_{x}=0.5$ is approximately 0.66 . The maximum $P_{c}$ obtained from the phase retarder is limited by the variation in the phase lag across the rocking curve $(\approx 0.96)$ with further reduction occurring due to the nonideal degree of linear polarization in the undulator beam $(\approx 0.95)$. The optics do not significantly affect the polarization of the EMW beam at these energies due to the low Bragg angles.

The figure of merit for each of these cases are shown in Figs. 13 and 14 normalized such that 1.0 corresponds to $1 \times 10^{12} \mathrm{ph} / \mathrm{s}$ with $P_{c}=1.0$. Fig. 13 shows that with $K_{x}=0.5$ the focused EMW is a much better source than the phase retarder, varying from 2.5 times better at $50 \mathrm{keV}$ to 2.0 times at $100 \mathrm{keV}$. The 
unfocused EMW beam is almost equivalent to the phase retarder for this $K_{x}$ value. For $\mathrm{K}_{\mathrm{x}}=1.0$, Fig. 14, the focused EMW and phase retarder are comparable with the phase retarder slightly better for energies above $67 \mathrm{keV}$. Note, however, that the single-bounce, meridinally focused monochromator provided the simplest focusing scheme for these energies. Kawata has recently constructed a double focusing monochromator capable of accepting the full EMW beam.[26] This can in theory increase the flux for the focused beam shown in Fig. 10 by a factor of $\approx 4$, doubling the figure of merit, although in practice only a factor of $\approx 2$ has been realized. Therefore, for cases in which polarization purity may be important, the phase retarder provides a nearly equivalent source to the EMW, but in general a focused EMW will be a better source than a phase retarder due to the higher figure of merit.

\section{Conclusions}

We have demonstrated that a phase retarder coupled with an undulator provides a more efficient source of circularly polarized $x$-rays for energies below $8.0 \mathrm{keV}$. This is especially true for $\mathrm{x}$-rays below $6 \mathrm{keV}$ where depolarization from the optics is important. Above $8.0 \mathrm{keV}$, a fully focused EMW beam can, in theory, provide a source $\sim 20 \%$ better than a phase retarder, but more likely the two techniques are roughly equivalent due to nonideal bending in the focusing crystal. For $x$-rays in the 50-100 keV energy range, the focused EMW' with $K_{x}=0.5$ provides a better source by approximately a factor of two for the focusing scheme compared, although this is accomplished by sacrificing some of the circular polarization.

We should note that, except for the meridinally focused crystal, this calculation compared essentially equal band passes for the EMW and phase retarder because the same set of crystal reflections was used. These reflections 
yielded the resolutions $\left(\Delta \mathrm{E} / \mathrm{E} \approx 4 \times 10^{-4}\right)$ necessary for resonant experiments in the low-energy regime and magnetic Compton measurements with approximately 0.05 A.U. resolution in the high-energy regime. Experiments that require a wider band pass, such as white-beam magnetic $x$-ray diffraction ${ }^{[27]}$ or energy dispersive techniques, would in general require an EMW. By proper dispersion matching, however, a transmission phase retarder can be used to simultaneously produce circularly polarized photons over a limited range $(150 \mathrm{eV}) \cdot{ }^{[9]}$

Thus, we have shown that phase retarders offer a viable alternative to EMWs for the production of circularly polarized $x$-rays in both energy regimes of interest for experiments probing magnetic phenomena. Furthermore this is accomplished while still retaining the freedom of not dedicating a beamline solely to experiments involving circular polarization.

\section{Appendix}

The figure of merit $\left(P_{c} \cdot \sqrt{I}\right)$ can be obtained by expressing the measured signal from a magnetic scattering or absorption experiment as a sum of terms arising from charge and magnetic effects,

$$
I^{ \pm} \sim \sigma_{\mathrm{c}} \mathrm{I}+\sigma_{\mathrm{m}} \mathrm{I}, \quad \sigma_{\mathrm{c}} \gg \sigma_{\mathrm{m}}
$$

Here $\mathrm{I}^{ \pm}$indicates the measured intensities taken with opposite helicities (or magnetizations), $I$ is the incoming beam intensity, and $\sigma_{\mathrm{c}}$ and $\sigma_{\mathrm{m}}$ are the charge and magnetic cross sections. For ferromagnetic materials, the magnetic cross section depends linearly on $\mathrm{P}_{c}$ (or the magnetization), ${ }^{[28-30]}$ thus can be separated out, $\sigma_{m} \sim P_{c} \sigma_{m}^{\prime}$, making the difference to sum ratio,

$$
\frac{I^{+}-I^{-}}{I^{+}+I^{-}} \sim \frac{2 P_{c} \sigma_{m}^{\prime} I}{2 \sigma_{c} I} \sim P_{c} \frac{\sigma_{m}^{\prime}}{\sigma_{c}} .
$$


Therefore the measured signal depends linearly on the degree of circular polarization. The percentage error in this quantity, which is the quantity to be minimized in any experimental measurement, is obtained by adding the errors in the numerator and denominator in quadrature,

$$
\left(\Delta \%\left(\frac{I^{+}-I^{-}}{I^{+}+I^{-}}\right)\right)^{2}=\left(\frac{\sqrt{I^{+}+I^{-}}}{I^{+}+I^{-}}\right)^{2}+\left(\frac{\sqrt{I^{+}+I^{-}}}{I^{+}-I^{-}}\right)^{2} .
$$

The first term above will always be much smaller than the second, thus can be neglected yielding,

$$
\Delta \%\left(\frac{I^{+}-I^{-}}{I^{+}+I^{-}}\right) \cong \frac{\sqrt{I^{+}+I^{-}}}{I^{+}-I^{-}} \sim \frac{\sqrt{2 \sigma_{c} I}}{2 P_{c} \sigma_{m}^{\prime} I} \sim \frac{1}{P_{c} \sqrt{I}} .
$$

Thus, the minimum error in the measurement is achieved by maximizing $P_{c} \cdot \sqrt{T}$. 


\section{References}

1. J.A. Golovchenko, B.M. Kincaid, R.A. Levesque, A.E. Meixner and D.R. Kaplan, Phys. Reo. Lett 57, 202 (1986).

2 D. Gibbs D.R. Harshman, E.D. Isaacs, D.B. McWhan, D. Mills, and C. Vettier, Phys. Reo. Lett. 61, 1241 (1988).

3 G. Schütz, M. Knülle, R. Wienke, W. Wilhelm, W. Wagner, P. Kienle, and R. Frahm, Z. Phys. B 73, 67 (1988).

4 E. Gluskin et al., IEEE Procedings (to be published) (1995).

5 P. Montano et al., Rev. Sci. Instrum. 66, 1839 (1995).

6 V.A. Belyakov and V.E. Dmitrienko, Sov. Phys. Usp. 32, 697 (1989).

7 K. Hirano, K. Izumi, T. Ishikawa, S. Annaka, and S. Kikuta, Jap. J. App. Phys. 30, L407 (1991).

8 K. Hirano, T. Ishikawa, S. Koreda, K. Fuchigami, K. Kanzaki, and S. Kikuta, Jpn. J. Appl. Phys. 31, L1209 (1992).

9 C. Giles, C. Malgrange, J. Goulon, F. de Bergevin, C. Vettier, E. Dartyge, A. Fontaine, C. Giorgetti, and S. Pizzini, J. Appl. Cryst. 27, 232 (1994).

10 J.C. Lang and G. Srajer, Rev. Sci. Instrum. 66, 1540 (1995).

11 B.W. Batterman, Phys. Rev. B 45, 12677 (1992).

12 S.D. Shastri, K.D. Finkelstein, Q. Shen, B.W. Batterman, and D.A. Walko, Rè. Sci. Instrum. 66, 1581 (1995).

13 D. M. Mills, Nucl. Istrum. Meth. A266, 531 (1988).

14 C.J. Yanke, G. Srajer, D.R. Haeffner, D.M. Mills, and L. Assoufid, Nucl. Istrum. Meth. A347, 128 (1994).

15 B.W. Batterman and H. Cole, Rev. Mod. Phys. 36, 681 (1964).

16 Calculated using code described in D.W. Berreman and A.T. Macrander, Phys. Rev. B 37, 6030 (1988). 
17 K. Hirano, T. Ishikawa, I. Nakmura, M. Mizutani, and S. Kikuta, Jpn. J. Appl. Phys. 33, L689 (1994).

18 J.C. Lang, S.D. Shastri, D.R. Haeffner, and G. Srajer, Argonne National Laboratory, (1995) (to be published).

19 B. Lai, A Khounsary, R. Savoy, L.R. Moog, and E. Gluskin, Argonne National Laboratory, ANL/APS/TB-3, (1993).

R.J. Dejus, B. Lai, L.R. Moog, and E. Gluskin, Argonne National Laboratory, ANL/APS/TB-17, (1994).

21 R.J. Dejus, Argonne National Laboratory, unpublished, (1995).

22 H. Kitamura and S. Yamamoto, Rev. Sci. Instrum. 63, 1104 (1992).

23 C. Malgrange, C. Carvalho, L. Braicovich, and J. Goulon, Nucl. Instrum. Meth. A308, 390 (1991).

24 T. Ishikawa, Rev. Sci. Instrum. 60, 2058 (1989).

25 S.D. Shastri, R.J. Dejus, D.R. Haeffner, and J.C. Lang, Argonne National Laboratory, (1995) (to be published).

26 H. Kawata, SPIE 1740, 130 (1992).

27 D. Laundy and S.P. Collins, Physica B 192, 50 (1993).

28 P.M. Platzman and N. Tzoar, Phys. Rev. B 2, 3556 (1970).

29 M. Blume and D. Gibbs, Phys. Rev. B 37, 1779 (1988).

30 J.P. Hannon, G.T. Trammell, M. Blume, and D. Gibbs, Phys. Reo. Lett. 61, 1245 (1988).

\section{DISCLAIMER}

This report was prepared as an account of work sponsored by an agency of the United States Government. Neither the United States Government nor any agency thereof, nor any of their employees, makes any warranty, express or implied, or assumes any legal liability or responsibility for the accuracy, completeness, or usefulness of any information, apparatus, product, or process disclosed, or represents that its use would not infringe privately owned rights. Referprocess disclosed, or represents to any specific commercial product, process, or service by trade name, trademark, manufacturer, or otherwise does not necessarily constitute or imply its endorsement, recommendation, or favoring by the United States Government or any agency thereof. The views and opinions of authors expressed herein do not necessarily state or reflect those of the United States Government or any agency thereof. 